DOI: https://doi.org/10.46296/yc.v5i9edespsoct.0114

\title{
USO CREATIVO DE LAS TICS EN EL DESARROLLO DE LAS DESTREZAS MATEMÁTICAS
}

\section{CREATIVE USE OF ICTS IN THE DEVELOPMENT OF MATHEMATICAL SKILLS}

\author{
Meza-Mendoza Yandri Gustavo ${ }^{1}$; Gallegos-Macías Marcos Ramón ${ }^{2}$ \\ ${ }^{1}$ Universidad San Gregorio de Portoviejo USGP. Portoviejo Ecuador. Correo: \\ e.ygmeza@sangregorio.edu.ec. ORCID ID: https://orcid.org/0000-0002-5212-8722 \\ ${ }^{2}$ Universidad San Gregorio de Portoviejo USGP. Portoviejo Ecuador. Correo: \\ mgallegos@sangregorio.edu.ec. ORCID ID: https://orcid.org/0000-0002-3651-034x
}

\section{Resumen}

Las tecnologías de la información y comunicación en los procesos de matemáticas juegan un rol crucial para que los estudiantes obtengan de forma oportuna las destrezas matemáticas, partiendo de ello se estableció como objetivo, determinar la influencia del uso de las TICs en el desarrollo de las destrezas matemáticas en los estudiantes de la básica superior. mediante la aplicación de procesos metodológicos, basados en un enfoque cuanti-cualitativo para la recolección de la información se utilizó la entrevista y encuesta por medio de lo que se estableció el uso creativo de las TICs relacionadas a las destrezas matemática tales como GeoGebra, Excel, Máxima, Meta Math, que ayudan a resolver problemas matemáticos. Se determina que las herramientas más usadas en la enseñanza de las matemáticas son plataformas virtuales y juegos digitales interactivos, así como la utilización de video tutoriales de la web, con la aplicación de estos recursos se evidencia que los estudiantes aumentaron su interés y motivación por las clases de matemáticas. De la misma manera es posible establecer que los docentes deben estar capacitados en la utilización de diferentes plataformas y herramientas TIC para el desarrollo de las destrezas matemáticas.

Palabras claves: Destrezas matemáticas; Entornos virtuales; Plataformas y herramientas TIC; Tecnologías de la Información y Comunicación.

\begin{abstract}
The information and communication technologies in mathematics processes play a crucial role for students to obtain mathematical skills in a timely manner. Based on this, the objective was established to determine the influence of the use of ICTs in the development of mathematical skills in high school students. Through the application of methodological processes, based on a quantitative-qualitative approach for the collection of information, an interview and survey were used to establish the creative use of ICTs related to mathematical skills such as GeoGebra, Excel, Maxima, Meta Math, which help to solve mathematical problems. It is determined that the most used tools in the teaching of mathematics are virtual platforms and interactive digital games, as well as the use of web video tutorials, with the application of these resources it is evident that students increased their interest and motivation for mathematics classes. In the same way, it is possible to establish that teachers should be trained in the use of different platforms and ICT tools for the development of mathematical skills.
\end{abstract}

Keywords: Mathematical skills; Virtual environments; ICT platforms and tools; Information and Communication Technologies.

Información del manuscrito:

Fecha de recepción: 22 de julio de 2021.

Fecha de aceptación: 24 de septiembre de 2021.

Fecha de publicación: 01 de octubre de 2021. 


\section{Introducción}

La ciencia y la tecnología se vuelve cada vez más exigentes e innovadora en los diferentes campos del mundo en que se vive. A raíz de ello es necesario que la comunidad educativa se actualice en el ámbito de las tecnologías de la información y comunicación, las cuales son fundamentales en el los procesos de enseñanza-aprendizaje de la actual sociedad (Carneiro, Díaz, y Toscano, 2021, p. 21) (Carneiro, Díaz, \& Toscano, 2021, pág. 21)

Es importante resaltar que la utilización creativa de las TICs en la enseñanza de las matemáticas, depende del esmero y dedicación del docente como también de los estudiantes para desarrollar las capacidades tecnológicas y a la vez potencializarlos a través de las herramientas que ofrece la internet. El uso de plataformas virtuales permite acceder a diversos $y$ extensos tipos de información que facilita la construcción de contenidos asociados para la formación del proceso de aprendizaje del área de matemáticas. (Grisales Aguirre, 2018, p. 11)
La UNESCO (2013), refiere que la inclusión de las TICs en América latina y el caribe sobre la educación tuvo un sin número de retos sin terminar, entre ellos; preparar eficazmente a los docentes, llevar a cabo programas de diseños curriculares, que permitan usar a gran escala las nuevas tecnologías (p. 6)

Avendaño (2015), expresa que, i2Indagando y apartando el impacto de las TIC en los logros de enseñanza y aprendizaje. En Ecuador el uso de las TICs se ha ido incrementando paulatinamente debido a la necesidad de las actualizaciones tecnológica educativa, dando paso a la transformación del conocimiento basado en el uso de las tecnologías de la información y comunicación. (p. 50)

Las tecnologías de la información y comunicación han supuesto un gran avance en el acceso de la información, sobre todo en el ámbito educativo, donde se experimentaron nuevos escenarios formativos que apostaron al intercambio de conocimiento inmediato entre docentes y estudiantes, permitiendo que se construyan nuevos 
aprendizajes en forma colaborativa, reflexiva y crítica, en un ambiente amigable, flexible, dinámico, pluripersonal y pluridimensional (MINEDUC, 2012, p. 12).

Guevara y Valencia (2020), mencionan además que el uso de las TICs, es un factor ineludible en la educación, siendo el constructivismo la esencia del aprendizaje en la actividad humana, para construir 0 crear conocimiento mediante la propia experiencia, las instituciones educativas han experimentado grandes cambios en el sistema de educación, en la incorporación de las TICs. (p.159)

En la provincia de Manabí del cantón Portoviejo de la zona noreste de la parroquia Andrés de Vera, del sector Medardo Cevallos, se encuentra ubicada la Unidad Educativa Francisco Pacheco, en el cual los estudiantes de la básica superior presentaron como principal problema un limitado uso creativo de las TIC, influyendo en el desarrollo de las destrezas matemáticas con un bajo rendimiento académico, produciendo el desinterés en los contenidos de aprendizaje, aburrimiento, la falta de práctica, la aglomeración de conocimientos teóricos que atribuyen en la disertación y a la vez, la perdida de año escolar.

La educación de hoy en día está basada en la era de la digitalización y tecnología, las TIC es una herramienta que permitió mejorar el proceso de aprendizaje mediante programas que facilitaron $y$ aportaron a una serie de beneficios, que ayudaron al desarrollo de las habilidades cognitivas de los estudiantes. Si bien es cierto, así como existió beneficios también tuvieron muchos retos y desafíos, como es el manejo y la cantidad de información que existe en internet

Este justificativo conllevo a conocer la realidad del escaso o limitado uso de las tecnologías de igual manera se pudo corregir el sistema tradicional para la mejora del conocimiento en las matemáticas e incluso se implementó y desarrollo metodologías y técnicas innovadoras que aportaron en resultados favorables a los alumnos y docentes de esta institución.

La forma y el grado de incluir las TICs dentro de la enseñanza de las matemáticas varía dependiendo del nivel educativo y los temas 
impartidos, ya que en cada situación proporcionan diversas formas de presentar situaciones idealizadas y problémicas, incitando al estudiante a desarrollar estrategias de resolución para su resolución, ayudándole a mejorar la comprensión de los conceptos matemáticos. Lo que implica que los docentes deben tener muy claro que las TICs no es solo un recurso o una herramienta didáctica de apoyo, sino que para poder incluirlas positivamente en el currículo de las matemáticas hay que redefinir las formas de cómo aprender y enseñar las matemáticas con ayuda de las TICs, debido a que se puede caer en el error de que lo que se está enseñando al estudiante es a manejar las herramientas tecnológicas y no los contenidos matemáticos (Guachun, 2016, p. 90) (Guachun, 2016, pág. 90)

La falta de capacitación y de conocimientos por parte del docente en el uso de las TIC, es uno de los factores que implica nuevos retos, pero sin duda se busca la forma y manera de llegar al estudiante para desarrollar en ellos, habilidades y destrezas que ayuden en el proceso de aprendizaje y comprensión de las matemáticas, respaldado por las Tecnologías de la Información y Comunicación, Por lo tanto se aclara que el uso de las herramientas TIC puede ser tomado como una herramienta didáctica predestinada a la solución de los contenidos de las ciencias exactas.

\section{Teoría y conceptos}

\section{Uso creativo de las TICs.}

Al no existir una definición conjunta, el uso creativo de las Tecnología de la Información y Comunicación, se lo ha desglosado en conceptos básicos de los mismos. Según (Aguilera, 2017) expresa "La creatividad es la habilidad para crear o inventar, en otras palabras, obtener de la nada algo desconocido "(p.3). La creatividad es la capacidad de generar nuevas ideas en búsqueda de soluciones de lo existente a lo trascendente.

Por otra parte, la definición de las TIC como lo indica (Maza, 2013) "Son aquellas tecnologías que permiten transmitir, procesar y difundir información de manera instantánea “(p. 1). Las TIC son un conjunto de recursos que permiten el acceso a múltiples informaciones de 
un sitio a otro mejorando las habilidades y destrezas de quienes la utilizan. Se precisa entonces que el uso creativo de las TIC, no es más que el desempeño creativo y propio que tiene cada docente al manejar de manera precisa esta herramienta tecnológica en los contenidos y destrezas a desarrollar.

Castillo (2020). "La educación por medio de entornos virtuales es el proceso de enseñanza aprendizaje en el que se produce una interacción alumno-profesor y este último sirve de guía al primero en un entorno virtual soportado en la red" (p. 33). Por su parte el alumno es totalmente autónomo y responsable de su aprendizaje.

Ahora bien, las redes telemáticas y el software permiten que este tipo de interacción se lleve a cabo de forma síncrona, y asíncrona. Cabe señalar que en la sincronía los alumnos y el profesor pueden reunirse en un chat o videoconferencia, salvando así las distancias geográficas.

"En una situación totalmente asíncrona, los alumnos y el profesor pueden interactuar intercambiando información en múltiples formatos, como foros, correo electrónico, wikis, webquest es decir a través de enlaces sin que se produzca concurrencia espacio temporal entre los participantes" (Espinoza Freire, Granda Ayabaca, \& Villacres Arias, 2020). En concreto el desarrollo de la educación se da en documentación electrónica, multimedia o impresa y la interactividad se da en un entorno de red.

En la actualidad como experiencias innovadoras en las destrezas matemáticas está el experimentar diversas vivencias vanguardistas, una de estas son el haber convertido nuestros hogares en un área de estudio donde se, incorporan recursos tecnológicos, dando paso a juegos dinámicos, retos y resoluciones de problemas matemáticos generando un aporte significativo a la comunidad educativa, que conlleva al fortalecimiento del entorno social.

\section{Destrezas matemáticas.}

Las destrezas matemáticas se relacionan con la capacidad y la habilidad para efectuar una tarea, con el fin de lograr un objetivo planteado. (Vidal, 2010) expresa "La competencia destreza en razonamiento matemático, consiste 
en desarrollar la comprensión matemática en los individuos a partir de niveles de razonamiento y fases de aprendizajes (p. 56).

El aprendizaje de las matemáticas se beneficia especialmente con el uso de las TIC, según (Lumbi, 2017) uno de ellos es la motivación, el interés, que es el impulso que le permite al estudiante aprender de forma agradable y entretenida a través de componentes lúdicos que los hace mucho más atractivos, enganchándolos y aumentando su capacidad cognitiva, en el proceso de aprendizaje, así como también la interactividad, la cooperación que es cuando el alumno comparte e intercambiar ideas fomentando a su vez, una comunicación mucho más estable creando estudiantes autónomos y capaces de tomar su propia decisión fortaleciendo la alfabetización digital y audiovisual en el uso de estas tecnologías.

En el proceso de aprender se utiliza una variedad de métodos idóneos y software educativos como, por ejemplo, GeoGebra, Excel, Máxima, Meta Math, Diedron, Calculadora matematicas, Math jump, Wiris, Demos, Buzzmath, Geometria dinámica entre otras estas son herramientas que ayudan al desarrollo de las destrezas matematicas, siendo esta una vía que conduce a la orientación de la enseñanza

Basándonos al método constructivista la cual el alumno construye sus propios conocimientos basados en la entrega de herramientas necesarias que le permite ser sujeto activos para así poder emitir juicios de valores que fomenten la transmisión y el condicionamiento de los conocimientos adquiridos.

\section{Metodología}

La investigación tuvo un enfoque cuanti-cualitativo que se utilizó con la finalidad de viabilizar el proceso de aplicación de instrumentos de recolección de información medible, misma que, posteriormente se tabuló para poder establecer la relación de las variables de estudio.

El tipo de investigación fue no experimental que Según Hernández, Fernández y Baptista. (2014) estableen que esta se realiza sin manipular las variables. Se centra en la observación de fenómenos tal y 
como se dan en su contexto natural.

Esta se aplicó en el estudio debido a que las variables únicamente serán medidas y estudiadas, pero estas no serán modificadas (p. 45).

Partiendo del método analítico, se analizaron las variables y sus componentes de forma teórica, mediante la recolección de información de textos especializados, relacionados con la temática tratada.

Las técnicas de investigación que se utilizaron fueron las encuestas $y$ entrevistas. La entrevista fue una técnica de gran utilidad en la investigación cuantitativa en la que se recabó datos; se definió como una conversación que se propuso un fin determinado distinto al simple hecho de conversar se aplicó a 8 especialistas del área de matemáticas y expertos en la utilización de las TICs.

La encuesta fue ampliamente utilizada como procedimiento de investigación, ya que permitió obtener y elaborar datos de modo rápido y eficaz. Esta técnica fue aplicada a los docentes de la institución y a los estudiantes.
Se consideró como población de estudio a los docentes de la Unidad Educativa Francisco Pacheco que imparten la materia de matemática en los diferentes niveles siendo un total de 14 docentes, y a los estudiantes de básica superior, siendo un total de 238, valor al que se le aplico fórmula de muestra finita, dio como resultado un total 147 estudiantes.

\section{Análisis de resultados y discusión}

\section{Análisis de los resultados}

Por medio del desarrollo de las aplicación de los instrumentos de encuestas fue posible conocer que el $36 \%$ de los docentes siempre priorizan que los estudiantes utilicen TIC para exponer o desarrollar procesos matemáticos en el entorno educativo; La aplicación de las TIC para el desarrollo de las matemáticas en el entorno educativo juega un rol esencial para el desarrollo de los aprendizajes, es por ello que los docentes deben de priorizar adecuadamente su aplicación en los procesos de enseñanza aprendizaje. Los autores Beltrán y Plascencia (2016) 
establecen que las TICs pueden ser benéficas en materia educativa, ya que brindan la posibilidad de extender el ámbito de estudio más allá de los límites físicos de la educación presencial (p. 1).

El $43 \%$ de los encuestados respondió que las herramientas tic que utilizan en el proceso de enseñanza aprendizaje de las matemáticas son las plataformas virtuales. Las herramientas tic en el proceso de enseñanza aprendizaje de las matemáticas juegan un papel esencial, pues, mediante las mismas los docentes pueden mediar el aprendizaje de forma didáctica propiciando una mejor abstracción de los conocimientos. "Las herramientas TIC facilitan la comprensión de las matemáticas ya que permiten modelar situaciones del mundo real; en la enseñanza de las matemáticas, el docente debe promover experiencias que permitan articular los contenidos." (Jiménez Daza, 2019, p. 3)

El $50 \%$ de los encuestados mencionaron que durante las clases de matemáticas sus estudiantes se han mostrado interesados y el $50 \%$ restante determino que se han presentado muy interesados. En el proceso de enseñanza y aprendizaje de las matemáticas es fundamental el contar con el interés del estudiante, ya que este permite un mejor entendimiento de los contenidos y los procesos matemáticos. "El docente debe intentar que los alumnos lleguen a apreciar el papel de las Matemáticas en la sociedad, sus campos de aplicación y su contribución al desarrollo social y cultural, por otro lado, hacerles comprender el funcionamiento del método matemático usándolo de manera ajustada a cada situación" (García Perales, 2016, p. 14)

El $71 \%$ de los encuestados expresaron que la inaccesibilidad al internet ha impedido alcanzar los objetivos de la materia; el $29 \%$ determino que el escaso equipo tecnológico ha propiciado que no se alcance los objetivos. En matemáticas contar con las herramientas didácticas y pedagógicas es un aspecto de suma importancia, porque de ello deriva la calidad del aprendizaje de los estudiantes. "Las dificultades de aprendizaje en matemáticas pueden ser una de las causas de fracaso 
escolar" (Fernández Carreira, 2013, p. 2)

El $93 \%$ los encuestados respondieron que pocos estudiantes han desarrollado las destrezas matemáticas que se plantearon como objetivos de la asignatura. Las destrezas matemáticas juegan un rol esencial en el proceso educativo de los estudiantes; el docente debe fomentar el adecuado desarrollo de las mismas durante el proceso de enseñanza aprendizaje. "La necesidad del conocimiento matemático crece día a día al igual que su aplicación en las más variadas profesiones y las destrezas más demandadas en los lugares de trabajo, son en el pensamiento matemático, crítico y en la resolución de problemas" (Ministerio de Educación, 2018, p. 1).

El $64 \%$ consideran que el aprovechamiento académico de sus estudiantes es poco satisfactorio. El aprovechamiento de los estudiantes es uno de los principales indicadores de falencias en los aprendizajes de allí la necesidad de aplicar estrategias para el mejoramiento de los conocimientos en el área de matemáticas. "El rendimiento académico escolar es una de las variables fundamental de la actividad docente, que actúa como halo de la calidad de un Sistema Educativo" (Jaspe, 2010, p. 1)

\section{Discusión}

Los datos obtenidos demuestran que el $36 \%$ de los docentes priorizan la utilización de las TICs para el desarrollo de procesos matemáticos, entre ellas la más empleada son las plataformas virtuales con un $43 \%$, y en el caso de los estudiantes, mocionan que se utilizan laptops; cuando los/as docentes utilizan TIC en el desarrollo de las clases, los estudiantes prestan un mayor interés a la misma, según el $50 \%$ de los docentes; a pesar de ello se presentan limitantes en la institución educativa, puesto que el $71 \%$ determino inaccesibilidad al internet, así como un escaso equipo tecnológico.

Según los autores Arévalo, et al. (2020) manifiesta que la no disponibilidad o lo obsoleto de los equipos que comúnmente tienen los estudiantes en sus hogares es una barrera para el uso de estas herramientas desde el hogar, lo que dificulta el ritmo y calidad de uso de las TIC y que a pesar de las 
limitantes técnicas el uso de las TIC es frecuente en la enseñanza de las matemáticas del cuerpo docente $(p$. 57). Por lo tanto, estas experiencias deben de enriquecerse con aplicaciones creativas como juegos interactivos y programas tales como hojas de cálculo, geogebra y drive, teams, zoom, skype, you tube, classroom, herramientas educativas multimedias como Educapley, Redaly; herramientas learning como Moodle y google classroom, libros o revistas digitales, software 0 aplicaciones online. De manera general es posible mencionar que los docentes, si aplican herramientas TIC para el desarrollo de las clases de matemáticas, pero las mismas son escasas, más que nada por la limitada capacidad de equipos tecnológicos de la institución la misma que se ve en la obligación de innovar y actualizar dichos recursos para un buen uso creativo de las TIC.

Respecto a la evaluación del desarrollo de las destrezas matemáticas que han logrado los estudiantes por medio de las TIC, es posible conocer que $79 \%$ de los docentes determinaron que son pocos los estudiantes que han desarrollado las destrezas matemáticas. Durante las clases los estudiantes casi siempre cumplen las actividades planteadas, según el $64 \%$ de los profesores; de la misma manera que se presentan muy interesados, según el $50 \%$ de los docentes, a pesar de ello el aprovechamiento académico de los estudiantes en las clases de matemáticas es poco satisfactorio según el $64 \%$ de los docentes.

Los autores Ríos y Yañez (2016) mencionan que "las competencias TIC que se relacionan con las habilidades de solución de problemas de matemáticas, son competencias que sirven de punto de partida para el desarrollo de otras habilidades y competencias en matemáticas; como el razonamiento, la comunicación" (p. 29). A partir de lo analizado es esencial que se desarrollen procesos orientados a mejorar las destrezas matemáticas por medio de la aplicación de las TIC, con la finalidad de mejorar el aprovechamiento de los educandos.

\section{Conclusiones}

El proceso e investigación llevado a cabo por medio de la aplicación de los instrumentos de investigación, 
fue posible establecer las siguientes conclusiones.

Al identificar las experiencias innovadoras en el uso creativo de las TICs relacionadas a las destrezas matemáticas por parte de los docentes es posible establecer que se prioriza la aplicación de las TIC; entre las más usadas se mencionaron plataformas virtuales, juegos digitales interactivos, videos, herramientas multimedias; con la aplicación de estos se ha logrado que los estudiantes aumenten su interés y motivación por las clases. A pesar de ello no se ha podido ampliar de manera oportuna la utilización de las TIC en las aulas de clases, especialmente por la inaccesibilidad del internet, así como, la existencia limitada de equipos tecnológicos en la institución.

En lo que refiere al desarrollo de las destrezas matemáticas que han logrado los estudiantes a través de las TICs se ha podido establecer que casi siempre se han cumplido las actividades planteadas para las clases, a pesar de ello se evidencia que el aprovechamiento de los estudiantes es en su mayoría poco satisfactorio.
Respecto a las recomendaciones didácticas los docentes deben de ser capacitados en la utilización de diferentes plataformas y herramientas TIC para el desarrollo de las destrezas matemáticas, entre las que se pueden mencionar Geogebra, Drive, Teams, zoom, skype, you tube, classroom; herramientas educativas multimedios como Educapley, Redaly; herramientas Learning como Moodle y google classroom, además de programas de creación de contenidos visuales, así como mensajería y videollamada.

\section{Bibliografía}

Aguilera, O. (2017). La creatividad del docente frente al uso de las TICs. Cuba: Monografia.com.

Arevalo Ribon, I. M., Corzo Camacho, M. A., \& Bordeth Meriño, J. (2020). Las tic como estrategia pedagógica en el fortalecimiento de la enseñanza aprendizaje de las matemáticas en el grado once de la institución departamental Alfonso López, municipio de San Sebastián, Magdalena. Universidad Cooperativa de Colombia. Recuperado el 4 de Abril de 2021, de 
https://repository.ucc.edu.co/ bitstream/20.500.12494/1806 6/3/2020_tic_estrategia_peda gogica.pdf

Avendaño Porras, V. d. (2015). Implementación y uso escolar de las tecnologías de la información y la comunicación: en la Meseta Comitéca Tojolabal del estado de Chiapas. Consejo Latinoamericano de Ciencias Sociales, p. 204. Obtenido de http://biblioteca.clacso.edu.ar/ Mexico/cresur/201611080610 00/TIC.pdf

Beltrán Cruz, A. d., \& Plascencia Cuevas, T. N. (2016). El uso de las TICs como herramienta de aprendizaje para alumnos de nivel superior. (U. A. Nayarit, Ed.) Recuperado el 13 de Mayo de 2021, de https://www.ecorfan.org/proc eedings/CDU_XI/TOMO\%20 11_2.pdf

Carneiro, R., Díaz, T., \& Toscano, J. C. (2021). Los desafíos de las TIC para el cambio educativo. Metas Educativas, p. 183. Recuperado el 13 de Mayo de 2021, de https://www.oei.es/uploads/fil es/microsites/28/140/lastic2.p df

Castillo López, D. (2020). Las TIC en los procesos de enseñanzaaprendizaje desarrollados por maestros tutores de Educación Primaria en la Región de Murcia. Revista
Interuniversitaria de Investigación en Tecnología Educativa. Recuperado el 2 de Abril de 2021, de https://revistas.um.es/riite/arti cle/view/432061/294771

Espinoza Freire, E. E., Granda Ayabaca, D. M., \& Villacres Arias, G. E. (2020). Influencia de las didácticas tecnológicas en el desarrollo del aprendizaje de los estudiantes (Vol. 3). Revista Metropolitana de Ciencias Aplicadas. Recuperado el 30 de Marzo de 2021, de https://remca.umet.edu.ec/ind ex.php/REMCA/article/view/3 11

Fernández Carreira, C. (2013). Principales dificultades en el aprendizaje de las Matemáticas. Pautas para maestros de Educación Primaria. UNIR. Recuperado el 14 de 2021 de Mayo, de https://reunir.unir.net/bitstrea $\mathrm{m} / \mathrm{handle} / 123456789 / 1588 / 2$ 013_02_04_TFM_ESTUDIO_ DEL_TRABAJO.pdf?sequenc $\mathrm{e}=1$

García Perales, R. (2016). Interés y motivación de los alumnos hacia las matemáticas: autopercepción de los más capaces (Vol. Vol. 3). Universidad de Castilla-La Mancha: Revista Internacional de Ciencia, Matemáticas y Tecnología. Recuperado el 14 de Mayo de 
2021

de

http://funes.uniandes.edu.co/

15389/1/Garc\%C3\%ADa201

6Inter\%C3\%A9s.pdf

Grisales Aguirre, A. M. (2018). Uso de recursos TIC en la enseñanza de las matemáticas: retos $\mathrm{y}$ perspectivas. $\quad$ p. 17. Recuperado el 14 de Mayo de 2021, de http://www.scielo.org.co/pdf/e ntra/v14n2/1900-3803-entra14-02-198.pdf

Guachun, F. (2016). Aplicacion e impacto de las TIC en las enseñanzas de las matematicas: "Una revision sistematica ". Cuenca: Universidad de Cuenca.

Guevara Vizcaíno, C. F., \& Valencia Velasco, F. K. (2020). Uso de las TIC en procesos de aprendizaje de matemática, en estudiantes de básica superior. Cuenca - Ecuador: Revista Cientifica: Dominio de las Ciencias. Obtenido de https://dominiodelasciencias. com/ojs/index.php/es/article/v iew/1279

Hernandez, Fernandez, \& Baptista. (2001). Metodología de la Investigación. Mexico: Editorial Mc Graw Hill.

Jaspe, C. (2010). Rendimiento Académico Escolar. Recuperado el 14 de Mayo de 2021, de http://wwwestrategias264.blo gspot.com/2010/07/rendimien to-academico-escolar.html

Jiménez Daza, D. A. (2019). Herramientas digitales para la enseñanza de las matemáticas en la educación básica. Universidad Cooperativa de Colombia. Recuperado el 14 de Mayo de 2021, de https://repository.ucc.edu.co/ bitstream/20.500.12494/1111 0/1/2019_herramientas_digita les_matematicas.pdf

Lumbi, S. (2017). Obtenido de DIALNET:

https://sites.google.com/site/a ulasticmatematicas/ventajasy-desventajas-de-las-aulastic-en-matematicas

Maza, G. (2013). Las TICen Ecuador. Quito: SlideShare.

MINEDUC. (2012). Tecnología de la información y la comunicación aplicadas a la educación. p. 12.

Ministerio de Educación. (2018). La importancia de enseñar y aprender matemáticas. Recuperado el 14 de 2021 de Mayo, de http://web.educacion.gob.ec/ _upload/10mo_anio_MATEM ATICA.pdf

Ríos Londoño, F. A., \& Yañez Figueroa, J. A. (2016). Las competencias tic y su relación con las habilidades para la solución de problemas de 
matemáticas. Revista

Electrónica de Tecnología

Educativa. ISSN 1135-9250.

Recuperado el 16 de Mayo de

2021,

de

file://C:/Users/PC/Downloads

/760-

Texto\%20del\%20art\%C3\%A

Dculo-2533-1-10-

20161031\%20(1).pdf

UNESCO. (2013). Enfoque sobre las

TICs en educación en

América Latina y El Caribe.

Oficina Regional de

Educación para América

Latina y el Caribe, p. 62.

Recuperado el 28 de Mayo de

2021,

de

http://www.unesco.org/new/fil

eadmin/MULTIMEDIA/FIELD/

Santiago/images/ticsesp.pdf

Vidal, L. (2010). Destrezas en razonamiento matemático. Biblioteca virtual Miguel de Cervantes, 1. Obtenido de http://www.cervantesvirtual.c om/obras/autor/vidalespinosa-leonidas-onesimo58066 